\title{
AFM tip-based cutting of grooves on permalloy nanowires for controlling the motion of magnetic domain walls
}

\author{
J. Jones ${ }^{1,2}$, E.B. Brousseau ${ }^{1}$, D. Read ${ }^{2}$ \\ ${ }^{1}$ School of Engineering, Cardiff University, Cardiff, United Kingdom \\ ${ }^{2}$ School of Physics and Astronomy, Cardiff University, Cardiff, United Kingdom
}

\begin{abstract}
The research reported in this paper presents a new application of the AFM tip-based nanomachining process in the context of investigating the development of data storage devices. In particular, nanoscale grooves are machined with the tip of an AFM probe on the top surface of nanowires made of a ferromagnetic material. These grooves are cut with the purpose of creating artificial pinning sites for controlling the motion of magnetic domain walls within such nanowires. The experimental and computational results obtained when implementing this approach on permalloy nanowires $55 \mathrm{~nm}$ thick and $320 \mathrm{~nm}$ wide show that the strength of such artificial pinning sites increases with the increase in the depth of the machined grooves. It is anticipated that the application of AFM tip-based nanomachining in this context could contribute to the development of a variety of magnetic domain wall devices including those for logic function, data storage or sensor applications.
\end{abstract}

Keywords: Atomic Force Microscope, Tip-based nanomachining, Magnetic nanowires, Magnetic domain walls.

\section{Introduction}

Nanoscale cutting performed with the tip of an Atomic Force Microscope (AFM) probe has been the subject of continued research investigations, which started only a few years after the original invention of the AFM [1]. Most implementations of this machining technique have been realised on standard AFM devices as in the studies reported in [2-5], or on modified commercial AFM platforms in order to overcome certain intrinsic drawbacks of AFM systems, such as their limited processing speed and area [6-10]. A few studies have also focussed on the development of customised machining set-ups for nanoscale cutting where the tool used was an AFM probe [11,12].

In addition, a number of research investigations implemented AFM tip-based nanomachining as part of a semiconductor-based process chain in order to develop devices for specific applications. Of particular interest in such studies is the fact that nanoscale physical phenomena, associated with the features cut with an AFM tip, were also characterised. For example, the fabrication of a single electron transistor on a GaAs/AIGaAs layered structure and its transport properties were reported in [13]. An antidot array was fabricated directly on InAs-AISb surface quantum wells by an oscillating tip and the magnetoresistivity of these structures were studied in [14]. A nanochannel connecting two micro-reservoirs was machined with an AFM tip in [15] and the flow of fluids and nanobeads through the channel was also demonstrated. The manufacture of a Ti nanowire was shown in [16]. In this case, AFM tip-based nanomachining was not used as a direct-write operation but rather as a patterning step prior to a lift-off process, which subsequently revealed the nanowire. Further fabrication and characterisation steps were conducted in this study to demonstrate the fabrication of a functional single titanium oxide nanodot for ultraviolet sensing. Finally, the research presented in [17] employed an array of heated tips to modify the topography of various polymer substrates with a view of developing a high density storage system, coined the "Millipede".
The research reported here is also concerned with demonstrating the potential of AFM tip-based nanomachining in the context of data storage applications. In particular, a possible technology that has attracted the attention of researchers in the past decade for the creation of memory logic and storage devices is based on the control of magnetic domain walls (DW) motions within nanowires [18-20]. The basic principle behind this technology is based on the fact that a ferromagnetic nanowire can contain regions, known as domains, which are magnetised in opposing directions. These domains can be used to represent the "ones" and "zeros" in binary code for information storage. Therefore, it is important to be able to control the motion of these magnetised regions via the controlled pinning of DWs. In comparison with current solid-state memories, such a magnetic DW-based technology exhibits the ability to read/write data at an increased rate and with symmetrical speed together with higher density storage. However, for magnetic DW-based devices to be successful, reliable and accurate control of the motions of the DWs is needed. In this paper, it is envisaged to implement AFM tipbased nanomachining for creating nanoscale grooves on the top surface of magnetic nanowires. More specifically, the function of grooves machined in this way is to act as pinning sites such that magnetic DWs are no longer able to freely move within the nanowires in very low externally applied magnetic fields.

In this context, this paper presents complementary experiments and simulations in order to study the influence of the depth of nano-grooves on the strength of DW pinning. To verify the computational results obtained, an AFM probe is used machine grooves across the width of nanowires in permalloy prior to conducting anisotropic magnetoresistance measurements for characterising the strength of the magnetic DW pinning. It has already been demonstrated in [21-22] that it is possible to cut grooves across the width of nanowires in permalloy with an AFM tip. However, the influence of the dimensions of grooves cut in this way on the pinning characteristic of magnetic DWs in such nanowires has not been reported previously.

World Congress on Micro and Nano Manufacturing

Edited by Joško Valentinčič, Martin Byung-Guk Jun, Kuniaki Dohda, and Stefan Dimov

Copyright (C) 2018 WCMNM 2018 Organisers :: Published by Research Publishing Singapore

ISBN: 978-981-11-2728-1 :: doi:10.3850/978-981-11-2728-1_44 


\section{Experimental methodology}

\subsection{Computational model}

Finite element-based micromagnetic simulations were carried out using the freely available ObjectOriented MicroMagnetic Framework (OOMMF) software [23]. The interactions between the elements in the model were governed by the Landau-Lifshitz-Gilbert equation of motion as follows:

$$
\frac{d \mathbf{M}}{d t}=-|\gamma| \mathbf{M} \times \mathbf{H}_{\mathrm{eff}}+\frac{\alpha}{M_{S}}\left(\mathbf{M} \times \frac{d \mathbf{M}}{d t}\right)
$$

where $\mathbf{M}$ is the magnetisation, $\mathbf{H}_{\mathrm{eff}}$ is the effective field, $\gamma$ is the Gilbert gyromagnetic ratio, $\alpha$ is the Gilbert damping parameter and $M_{s}$ is the saturation magnetisation.

The Euler method was used to solve the equation and thus, to determine the relative pinning position of DWs as the externally applied magnetic field is sequentially increased. The length and width of each cell in the model was $5 \mathrm{~nm}$ while the thickness of a cell varied depending on the overall thickness of the nanowire, with $5 \mathrm{~nm}$ being the maximum cell thickness utilised in order to remain below the expected exchange length. A nanoscale groove was also simulated by setting a region of the mesh, in the centre of the top surface of modelled nanowires, with the parameters of a vacuum. A DW was also positioned beneath the groove to initialise each simulation. Upon starting the simulation, the system was allowed to relax before applying a stepwise increasing external magnetic field to find the point at which the DW depinned. The material parameters used for permalloy are given in Table 1. In addition, the Gilbert damping parameter, $\alpha$, was set to 0.5 , as is commonly adopted for such calculations to improve the speed of the simulations. Fig. 1 illustrates the model set-up prior to the initial relaxation step for a given groove depth and width.

\subsection{Nanowire fabrication}

The permalloy source material used in this study had an approximate $80 \%$ nickel and $20 \%$ iron content. The nanowires were fabricated using typical lithographic processing techniques, which are illustrated in Fig. 2 and described as follows. A PMMA resist was first spin-coated on a silicon substrate. Electron beam lithography was then employed to expose the resist in a pattern that defined the overall lateral shape of the nanowires. A standard developer and oxygen plasma ash were subsequently applied to remove the exposed PMMA. Next, thermal evaporation was performed to deposit permalloy prior to the last step consisting of a standard lift-off procedure to remove the remaining resist and reveal the final

Table 1

Permalloy properties defined for OOMMF simulations

\begin{tabular}{ll}
\hline Parameter & Value \\
\hline Saturation magnetisation $\left(M_{s}\right)$ & $8.10^{5} \mathrm{~A} / \mathrm{m}$ \\
Exchange constant $(A)$ & $1.3 .10^{-11} \mathrm{~J} / \mathrm{m}$ \\
Anisotropy constant $(K)$ & $0 \mathrm{~J} / \mathrm{m}^{3}$ \\
\hline
\end{tabular}

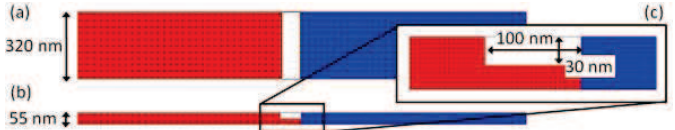

Fig. 1. Example of a modelled nanowire with a groove, 100 $\mathrm{nm}$ wide and $30 \mathrm{~nm}$ deep, on its top surface, upon setting-up the initial magnetisation conditions: (a) top view, (b) side view and (c) zoomed-in view of the groove.

permalloy nanowire design. The nanowires produced in this way were $55 \mathrm{~nm}$ thick and $320 \mathrm{~nm}$ wide.

\subsection{Cutting of nanoscale grooves}

The subsequent cutting of nanoscale grooves on the surface of the nanowires was realised on a commercial AFM system (XE-100 from Park Systems), using probes with a four-sided diamond tip with a radius quoted as less than $50 \mathrm{~nm}$ (ND-DYCRS from Advanced Diamond Technologies). These probes exhibited a nominal normal spring constant of $35 \mathrm{~N} / \mathrm{m}$. The actual value of this spring constant was determined experimentally by following a well-known method for rectangular cantilevers [24]. Initial experiments were conducted on thin films of the same thickness of the nanowires to be tested. Applying varying forces to cut into the surface revealed that a minimum load of $1 \mu \mathrm{N}$ was required to initiate groove formation on the nanowires.

\subsection{Anisotropic magnetoresistance measurements}

Four-terminal anisotropic magnetoresistance (AMR) measurements were carried out to determine the DW pinning strength of nanoscale grooves in the magnetic nanowires. In a four terminal AMR measurement, two of the terminals are utilised for the flow of current through the nanowire, while the other

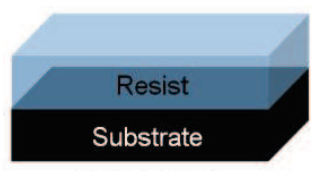

1. Spin coat

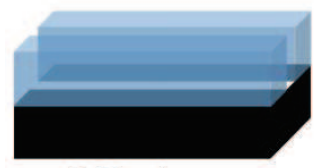

3. Develop

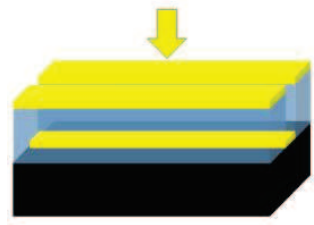

5. Deposit

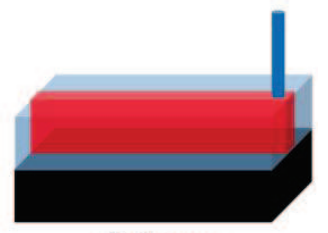

2. Expose

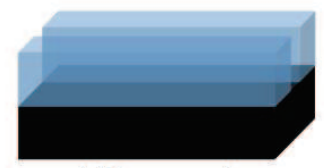

4. Plasma ash

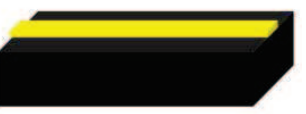

6. Lift-off
Fig. 2. Illustration of the process chain used to generate permalloy nanowires. 
two act as voltage probes to monitor the electrical resistance in the region of interest within the nanowire. These measurements are conducted under the influence of an external magnetic field. This electrical resistance measured depends on the angle between the current and the magnetisation direction of the material. In the configuration where the external applied magnetic field and the current density are parallel or anti-parallel, as was the case in this study, a decrease in the measured resistance shows the presence of a DW. The value of the external applied magnetic field subsequently required to depin the DW is taken to represent the strength for the pinning site under evaluation.

\section{Results and discussion}

Fig. 3 summarises the measured experimental results. In particular, the strength of the externally applied magnetic field required to remove a DW from the artificially created pinning site is given as a function of the depth of the machined groove. This figure also shows the topography of the groove machined at each experimental data point. This topography was obtained using the AFM instrument in non-contact mode with a different type of probe designed for imaging purpose (model NSG30 from NT-MDT). It can be seen from this figure that the pinning strength increases with the increase in the groove depth. Notches and grooves in a magnetic nanowire modify the potential energy landscape and can lead to a local increase in the field required to propagate a DW along the wire. The exact micromagnetic configuration of the DW relative to the shape of the groove in a wire can lead to a complex combination of energy maxima or minima trapping DWs, a phenomena that has been extensively studied in laterally notched wires [25].

Fig. 4 shows an example of a simulated DW pinned at the location of a groove. Such a DW is known as a vortex wall. Given the dimensions of the modelled nanowire, it was expected that the simulated DW would be of the vortex type rather than of the transverse type [26]. In addition, the plot given in Fig. 5 provides a comparison between the measured and the simulated pinning strength of the nanoscale grooves on permalloy nanowires. It can be seen that the experimental and simulated results follow the same trend, i.e. that the increase in groove depth results in an increase in the pinning strength. A difference between the simulation and experimental results with respect to the actual value of the pinning strength can also be observed from this plot. This is due to the fact that the simulations are carried out at zero

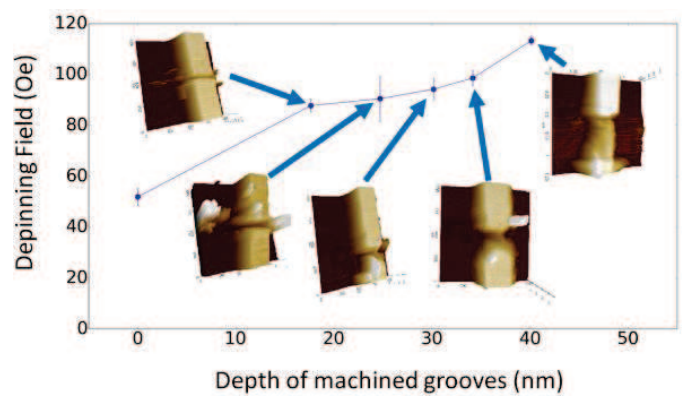

Fig. 3. Plot of the required depinning field as a function of the depth of grooves machined on the nanowire.

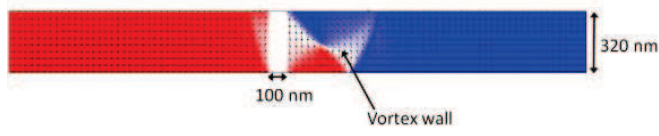

Fig. 4. An example of an initial relaxed state of the simulations - top view of the nanowire.

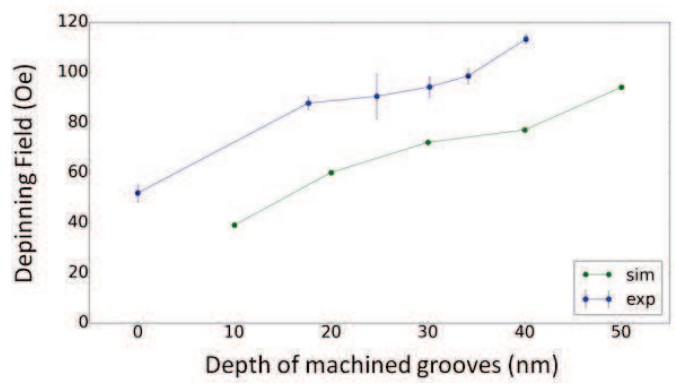

Fig. 5. Comparison between the experimentally measured and simulated pinning strengths of grooves on permalloy nanowires.

temperature, while the experiments were conducted at finite room temperature.

The results presented here are in-line with those reported by Narayanapillai and Yang [27]. However, these authors focussed mainly on a computational study. Thus, the data obtained in this work also provide an experimental confirmation of previous findings.

\section{Conclusions}

The paper reports on a new application of the AFM tip-based nanomachining process. In particular, nanoscale grooves are machined in this way on the top surface of permalloy nanowires to create artificial pinning sites for controlling the motion of magnetic domain walls. It is anticipated that this technique could contribute to the development of a variety of magnetic DW devices including those for logic function, data storage or sensor applications.

The experimental and computational results obtained with permalloy nanowires $55 \mathrm{~nm}$ thick and $320 \mathrm{~nm}$ wide show that the strength of such artificial pinning sites increases with the increase in the depth of the machined grooves.

\section{Acknowledgements}

The reported research was funded by the Engineering and Physical Sciences Research Council (EPSRC) under the grant EP/M020703/1.

\section{References}

[1] G. Binnig et al., "Atomic Force Microscope," Phys. Rev. Lett., 1986; 56(9), pp. 930-934.

[2] T.H. Fang et al., "Machining characterization of the nano-lithography process using atomic force microscopy," Nanotechnology, 2000; 11, pp. 181-187. [3] J.A. Blach et al., "A mechanistic approach to tipinduced nano-lithography of polymer surfaces," Thin Solid Films, 2004; 459, pp. 95-99.

[4] J.E. Headrick et al., "Nanoscale patterning of alkyl monolayers on silicon using the atomic force microscope," Langmuir, 2005; 21, pp. 4117-4122. 
[5] H.D. Fonseca Filho et al., "Metal layer mask patterning by force microscopy lithography," Mat. Sci. and Eng. B, 2004; 112, pp. 194-199.

[6] Y.D. Yan et al., "Investigation on AFM-based micro/nano-CNC machining system," Int. J. Machine Tools \& Manuf., 2007; 47, pp. 1651-1659.

[7] L. Zhang et al., "High-rate tunable ultrasonic force regulated nanomachining lithography with an atomic force microscope," Nanotechnology, 2012; 23: 085303 (9 pages).

[8] S. Park et al., "Vibration assisted nano mechanical machining using AFM probe," CIRP Annals - Manuf. Tech., 2014; 63, pp. 537-540.

[9] E.B. Brousseau et al., "A hybrid roll-to-roll AFM setup for high throughput tip-based nano-machining," Manuf. Lett., 2015; 6, pp. 10-13.

[10] Geng Y. et al., "AFM tip-based nanomachining with increased cutting speed at the tool-workpiece interface," Prec. Eng., 2018; 51, pp. 536-544.

[11] K. Bourne et al., "Study of a high performance AFM probe-based microscribing process," J. Manuf. Sci. and Eng., 2010; 132(3), 030906 (10 pages).

[12] B.A. Gozen et al., "Design and evaluation of a mechanical nanomanufacturing system for nanomilling," Prec. Eng., 2012; 36(1), pp. 19-30.

[13] H.W. Schumacher et al., "Controlled mechanical AFM machining of two-dimensional electron systems: fabrication of a single electron transistor," Physica E, 2000; 6(1-4), pp. 860-863.

[14] J. Cortes Rosa et al., "Direct patterning of surface quantum wells with an atomic force microscope," Appl. Phys. Lett., 1998; 73(18), pp. 2684-2686.

[15] Z. Wang et al., "Nanochannel system fabricated by MEMS microfabrication and atomic force microscopy," IET Nanobiotechnology, 2011; 5(4), pp. 108-113.

[16] L.-Y. Hong et al., "Fabrication of single titanium oxide nanodot ultraviolet sensors by atomic force microscopy nanolithography," Sensors and Actuators A: Physical, 2015; 232, pp. 94-98.

[17] P. Vettiger et al., "The "Millipede"- Nanotechnology entering data storage," IEEE Trans. Nanotech., 2002; 1(1), pp. 39-55.

[18] M. Hayashi et al., "Current-controlled magnetic domain-wall nanowire shift register," Science, 2008; 320, pp. 209-211.

[19] D. Allwood et al., "Magnetic domain-wall logic," Science, 2005; 309, pp. 1688-1692.

[20] S.S.P. Parkin et al. "Magnetic domain-wall racetrack memory," Science, 2008; 320, pp. 190-194. [21] A.A. Tseng et al., "Nanomachining of permalloy for fabricating nanoscale ferromagnetic structures using atomic force microscopy." J. Nanosci. and Nanotech., 2010; 10(1), pp. 456-466.

[22] A.A. Tseng et al., "Scratching properties of nickeliron thin film and silicon using atomic force microscopy," J. Appl. Phys., 2009; 106(4), 44314.

[23] The Object Oriented MicroMagnetic Framework (OOMMF) project at ITL/NIST. Code available at http://math.nist.gov/oommf (website last accessed on 21/03/2018).

[24] J.E. Sader et al., "Calibration of rectangular atomic force microscope cantilevers," Rev. Sci Instr., 1999; 70(10), pp. 3967-3969.

[25] D. Petit et al., "Domain wall pinning and potential landscapes created by constrictions and protrusions in ferromagnetic nanowires," J. App. Phys., 2008; 103, 114307.

[26] Y. Nakatani, et al., "Head-to-head domain walls in soft nano-strips: A refined phase diagram," Journal of
Magnetism and Magnetic Materials, 2005; 290-291, pp. 750-753.

[27] K. Narayanapillai et al., "Control of domain wall motion at vertically etched nanotrench in ferromagnetic nanowires," Applied Physics Letters, 2013; 103(25), pp. 2011-2015. 